\title{
Optical Gain from InAs Nanocrystal Quantum Dots in a Polymer Matrix
}

\author{
Gang Chen, ${ }^{1}$ Ronen Rapaport, ${ }^{1}$ Dan T. Fuchs, ${ }^{1}$ \\ Sahar Vilan, ${ }^{1}$ Assaf Aharoni, ${ }^{2}$ and Uri Banin ${ }^{2}$ \\ ${ }^{1}$ Bell Laboratories, Lucent Technologies, \\ 600 Mountain Avenue, Murray Hill, New Jersey 07974 \\ ${ }^{2}$ Department of Physical Chemistry and the Farkas Center for Light Induced Processes, \\ The Hebrew University, Jerusalem 91904, Israel
}

\begin{abstract}
We report on the first observation of optical gain from InAs nanocrystal quantum dots emitting at 1.55 microns based on a three-beam, time resolved pump-probe technique. The nanocrystals were embedded into a transparent polymer matrix platform suitable for the fabrication of integrated photonic devices.
\end{abstract}


The recent development of chemically synthesized nanocrystal quantum dots (NQDs) with widely tunable emission color ${ }^{\underline{\underline{1}}}$ has greatly broadened the scope of the future application of quantum dots in lasers and amplifiers. Optical gain and amplified stimulated emission from NQDs has already been observed both in the visible using $\mathrm{CdSe}^{2.3}$ and nearIR using $\mathrm{PbSe}^{\underline{4}}$ as the core material. A different system studied for near-IR (around $1.5 \mu \mathrm{m}$ telecommunication wavelength) applications uses InAs $\stackrel{5.6}{ }$, which, compared to PbSe, has a much lower degree of degeneracy for the lowest quantized states ${ }^{\underline{T}}$. It could therefore be a promising candidate for reducing gain threshold.

It is also important to find a convenient way to integrate NQDs into future planar optical circuits. We have recently developed a low-loss polymer platform in which the InAs as well as PbSe NQDs were dispersed into perfluorocyclobutane (PFCB) polymer with their optical

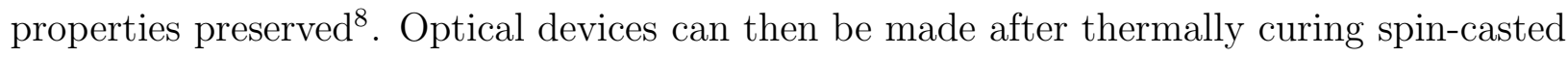
NQD-polymer films $\stackrel{8}{*}$. Here we present the first experimental evidence for the observation of optical gain in an InAs-NQD-polymer film ready for device fabrication at room temperature. Using a two- and a three-beam time-resolved pump-probe techniques, we measure the gain dynamics of the InAs NQDs, extracting their gain lifetime and the gain recovery time.

$\mathrm{PFCB}^{9}$ films containing InAs NQDs were fabricated following the procedure described in Ref. $\underline{\underline{8}}$. The NQD weight fraction in the polymer host was experimentally measured to be $1 \%$ (based on optical technique mentioned in Ref. $\underline{\underline{8}}$ ), corresponding to an InAs volume fraction of $0.27 \%$. For an average NQD size of $8 \mathrm{~nm}$ (estimated from TEM images), it corresponds to an NQD density of $1.0 \times 10^{16} \mathrm{~cm}^{-3}$. The Film thickness was measured to be $0.6 \mathrm{~mm}$. Typical absorption and photoluminescence (PL) spectra at room temperature are shown in Fig. 1(a) and are not affected by the NQD-polymer mixing process ${ }^{8}$.

The optical measurements were performed at room temperature and were based on a three-beam pump-probe technique $\underline{\underline{\underline{10}}}$ schematically shown in Fig. 1(b). The optical pulses ( $\sim 120 \mathrm{fs})$ were generated using two independently wavelength tunable optical parametric amplifiers in the near IR pumped by a single $800 \mathrm{~nm}$ Ti:Sapphire regenerative amplifier. The optical pulses generated from the first OPA were used as a pre-pump. The pulses from the second OPA were spit into a pump and a probe beam. The time delays between each of the three light pulses were controlled using two motorized delay stages. The three beams were focused on the film using long focal length lenses such that their overlap length is comparable to the sample thickness. In a typical measurement, the probe intensity was 


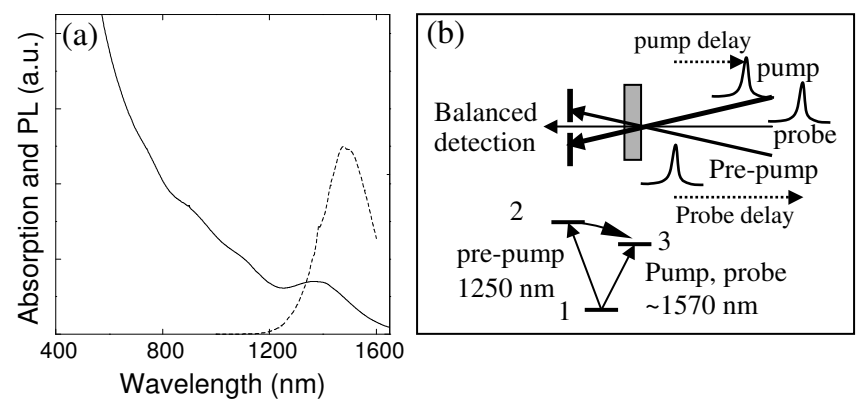

FIG. 1: (a) Absorption (solid line) and photoluminescence (dashed line) spectra of the InAs NQDpolymer film. (b) A schematic diagram of the three-beam pump-probe setup and the level diagram for the NQDs. For the conventional two-beam pump-probe measurements, the resonant pump beam is blocked.

maintained extremely low.

This technique offers a convenient way to measure small internal optical gain even in the presence of appreciable device losses. Here, the pre-pump is tuned to $1250 \mathrm{~nm}$ and excites the NQDs from the ground state (level 1 of Fig. 1 (b)) to higher excited states (level 2). The excited carriers then quickly relax nonradiatively to the lowest confined levels of NQDs (level 3) to possibly give rise to a population inversion. The pump and probe are tuned to be resonant with the transition between the level 1 and level 3 at $1570 \mathrm{~nm}$ and pass through the sample shortly after the excitation by the pre-pump. The differential transmission of the probe due to the resonant pump is measured. In the case that no population inversion between level 3 and 1 is induced by the pre-pump, the probe experiences saturated absorption without the resonant pump. Because the resonant pump excites even more carriers to level 3 , the probe experience even less absorption due to the saturation of the $3-1$ transition. The differential probe transmission signal in this case would be positive following the resonant pump. When there is population inversion, however, the probe experiences a gain without the resonant pump. The presence of the resonant pump would only reduce the population inversion via stimulated emission, leading to less probe gain. The differential probe transmission signal in this case is therefore negative immediately after the resonant pump. Thus, by studying the sign of such a differential signal, one can unambiguously determine whether the NQDs are population inverted, even in the presence of strong losses.

Fig. 2 (a) presents the probe transmission traces as a function of the delay between 
the probe and the pre-pump for various pre-pump fluences (in units of $\mathrm{mJ} / \mathrm{cm}^{2}$, fluence is for each pulse) in the common two-beam pump-probe experimental geometry (with the resonant pump blocked). The signals are normalized to their values when the pre-pump fluence is zero. These traces show that after the excitation by the pre-pump, level 3 is quickly populated via nonradiative relaxation of carriers from level 2 to 3 , and the probe experiences an increase in transmission, as expected. As the pre-pump intensity is turned up, the level 3 population following the pre-pump increases and so does the probe transmission signal. At low pre-pump excitation intensity, the probe transmission after the excitation of the pre-pump follows a single exponential decay with a decay constant of $\sim 300$ ps (carrier radiative lifetime), shown by the curve fitting for traces with pre-pump fluences equal to or smaller than $0.4 \mathrm{~mJ} / \mathrm{cm}^{2}$. Ideally, this corresponds to an excitation of less than an average of $1 \mathrm{e}-\mathrm{h}$ pair per $\mathrm{NQD}^{2}$. Indeed, the number of excited e-h pairs for $0.4 \mathrm{~mJ} / \mathrm{cm}^{2}$ pre-pump is estimated to be 0.8 , using an NQD density of $1.0 \times 10^{16} \mathrm{~cm}^{-3}$ (see above), a measured beam size of $100 \mu \mathrm{m}$ and a measured pre-pump absorption of 30\%. For higher pre-pump excitation fluences that excites more than 1 e-h pairs per NQD, the probe transmission decay clearly becomes multi-exponential with the faster components determined by the enhanced nonradiative Auger relaxation 11 .

Fig. 2 (b) presents the probe transmission traces as a function of the delay between the probe and the pre-pump and as a function of the pre-pump fluence for the three-beam measurements. The resonant pump is delayed by 5ps (marked by the arrow) and is maintained a constant intensity (fluence of $1.2 \mathrm{~mJ} / \mathrm{cm}^{2}$ ). The probe transmission is normalized to its value when all pumps are off. The dark lines represent traces where the resonant pump is off, similar to the two-beam pump-probe experiments discussed previously. In these measurements, whether the population between level 1 and 3 is inverted is determined by the change of the probe transmission immediately after the excitation by the resonant pump. At low pre-pump intensity, level 3 is less populated than level 1, the resonant pump thus drives level 3 occupancy higher, leading to increased transmission of the probe. This shows up as an additional peak on top of the probe transmission traces at 5 ps where the resonant pump arrives. At high pre-pump intensity, however, level 3 and 1 population is inverted. The resonant pump reduces the inversion via stimulated emission. The probe thus experiences less gain and its transmission dips at the arrival of the resonant pump. The dotted line in Fig. 2 (b) marks the probe transmission level where the dip and peak tend to meet. It 

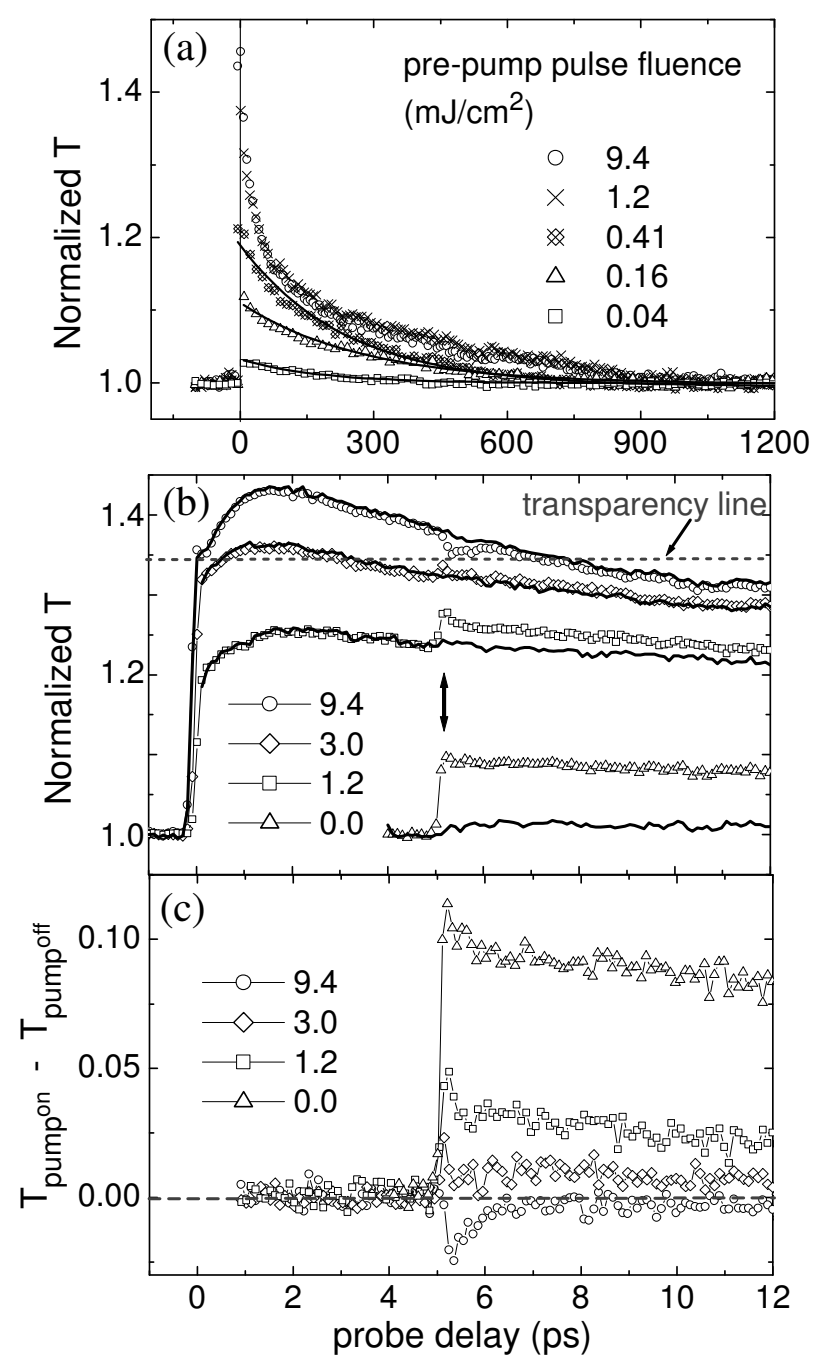

FIG. 2: (a) Normalized probe transmission as a function of the pre-pump - probe delay for different pre-pump fluences in the two-beam geometry in which the resonant pump is not present. (b) Same as (a), but now the resonant pump pulse is used and is delayed by 5ps with respect to the prepump (marked by the arrow). The dark lines present the corresponding two-beam pump-probe experimental traces with the resonant pump off. The dashed line (transparency line) marks the onset of gain. (c) Same data as in (b) presented as differential probe transmission due to the resonant pump.

represents the transparency level of the probe.

The differential probe transmission due to the resonant pump (the symboled traces in Fig. 2 (b) minus the corresponding dark line traces) is plotted in Fig. 2 (c), showing the sign 

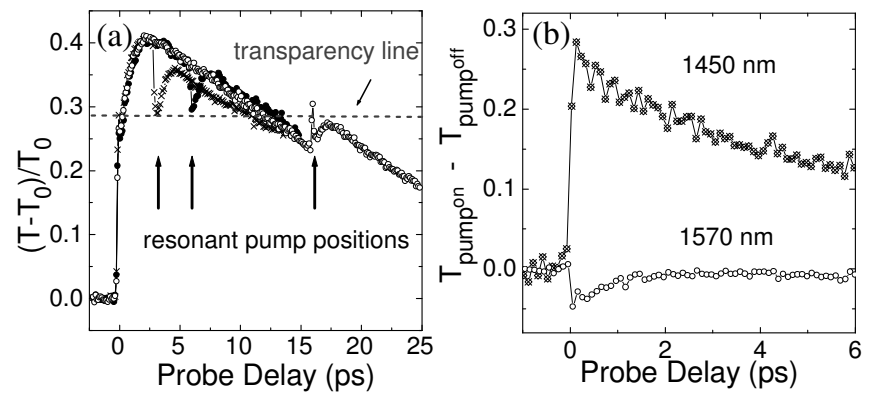

FIG. 3: (a) Normalized probe transmission with different resonant pump delays, marked by the arrows. The dashed line (transparency line) marks the onset of gain. The pre-pump fluence is $9.4 \mathrm{~mJ} / \mathrm{cm}^{2}$. (b) Probe Differential transmission for two different probe and resonant pump wavelengths with a pre-pump fluence of $9.4 \mathrm{~mJ} / \mathrm{cm}^{2}$ and wavelength of $1250 \mathrm{~nm}$.

change between the gain and no-gain cases. The sign change occurs roughly at a pre-pump fluence of $3 \mathrm{~mJ} / \mathrm{cm}^{2}$, with 15\% absorbed by the NQD film (measured), corresponding to an excitation of 6 e-h pairs per NQD (see above for parameters used). Since the gain threshold occurs at 1 e-h pair at the lowest confined levels per NQD, this suggests that the quantum efficiency of exciting electrons into the lowest confined states by the pre-pump is about $20 \%$. The maximum internal gain achieved in these measurements was $2 / \mathrm{cm}$, which corresponds to an NQD gain cross section of $2 \times 10^{-16} \mathrm{~cm}^{2}$ for a NQD density of $1.0 \times 10^{16} \mathrm{~cm}^{-3}$. We note that much higher gain is expected by decompressing the pre-pump pulse to increase excitation efficiency, by improving the NQD quantum efficiency, and by increasing the NQD loading in the polymer host.

The measurements above show that immediately after the excitation by a strong prepump, level 3-1 population is inverted. The carrier decay in the NQDs, however, leads to a reduction and eventually loss of the population inversion after the pre-pump passes the sample. By delaying the resonant pump and probe pulses different amount of time relative to the pre-pump, the gain lifetime can be measured. This is shown by the probe transmission signal in Fig. 3 (a) for a pre-pump and pump fluence of 9.4 and $3.0 \mathrm{~mJ} / \mathrm{cm}^{2}$ respectively. The arrows indicate the position of the resonant pump, For short resonant pump delay times, a negative differential signal of the probe transmission is observed, indicating that the probe experiences an internal gain. At longer delays, the differential signal is positive, which indicates that the population inversion and gain is lost. The probe transmission tends 
to approach the transparency line (doted line) immediately after the resonant pump for all pre-pump-pump delays. From the crossing between the decay curves and the transparency line, the gain lifetime can be inferred to be $\sim 10$ ps. The short gain lifetime corresponds to the fast initial carrier decay when the NQDs are occupied by more than 1 e-h pairs. The dominant source for this decay is likely to be the nonradiative Auger recombination $\frac{11}{}$.

The carrier dynamics after the excitation by the resonant pump can be extracted from Fig. 2 (c). For low pre-pump intensities (no-gain regime), the positive differential probe transmission signal decay similar to that of the high power two-beam pump-probe signal, with the decay rate determined by the Auger as well as the radiative carrier recombination. For high pre-pump intensities (gain regime), the negative differential probe transmission signal recovers on a time scale of $\sim 2 \mathrm{ps}$, much faster than the carrier recombination time. In this case, the resonant pump first reduces the level 3 population via stimulated emission, effectively depleting the probe gain instantaneously. The probe gain then recovers. This gain recovery can be explained by other highly excited electrons quickly replenishing the NQDs depleted by the resonant pump to recover the level 3 occupancy. Gain recovery was also observed in other systems and its time scale is a very important parameter lasers and amplifiers.

Finally, we show in Fig. [3 (b) traces of the differential probe transmission as a function of the probe-pre-pump delay under the same high pre-pump fluence $\left(9.4 \mathrm{~mJ} / \mathrm{cm}^{2}\right)$ for two different wavelengths of the probe and the resonant pump. Again, the resonant pump is delayed by $5 \mathrm{ps}$ with respect to the pre-pump. While at $1570 \mathrm{~nm}$ the probe experiences gain, it does not experience any net gain and shows a positive differential signal at $1450 \mathrm{~nm}$. This confirms that population first occurs at the low energy side of the inhomogeneously broadened spectrum of the NQD ensemble.

In summary, we have successfully incorporated InAs NQDs emitting at $1.55 \mu \mathrm{m}$ into a PFCB polymer platform. We show for the first time optically induced population inversion and gain from an PFCB film containing InAs NQDs based on a three-beam pump-probe technique. The gain lifetime and recovery time were studied. The authors thank Xing Wei 
and Phil Platzman for helpful discussions.

1 C. B. Murray, S. Sun, W. Gaschler, H. Doyle, T. A. Betley, and C. R. Kagan, IBM J. Res. and Dev. 45, 47 (2001).

2 V. I. Klimov, A. A. Mikhailovsky, S. Xu, A. Malko, J. A. Hollingsworth, C. A. Leatherdale, H. J. Eisler, and M. G. Bawendi, Science 290, 314 (2000).

3 H. J. Eisler, V. C. Sundar, M. G. Bawendi, M. Walsh, H. I. Smith, and V. Klimov, Appl. Phys. Lett. 80, 4614 (2002).

4 R. D. Schaller, M. A. Petruska, and V. I. Klimov, J. Phys. Chem. B 107, 13765 (2003).

5 Y. W. Cao and U. Banin, J. Am. Chem. Soc. 122, 9692 (2000).

6 A. A. Guzelian, U. Banin, A. V. Kadavanich, X. Peng, and A. P. Alivisatos, Appl. Phys. Lett. 69, $1432(1996)$.

7 I. Kang and F. W. Wise, J. Phys. Chem. B 14, 1632 (1997).

8 Y. K. Olsson, G. Chen, R. Rapaport, D. T. Fuchs, V. C. Sundar, J. S. Steckel, M. G. Bawendi, A. Aharoni, and U. Banin, Appl. Phys. Lett. 85, 4469 (2004).

9 J. D. W. Smith, S. Chen, S. M. Kumar, J. Ballato, C. Topping, and S. H. Foulger, Adv. Mater. 14, 1585 (2002).

10 K. Kim, J. Urayama, T. B. Norris, J. Singh, J. Phillips, , and P. Bhattacharya, Appl. Phys. Lett. 81, 670 (2002).

11 V. I. Klimov, A. A. Mikhailovsky, D. W. MacBranch, C. A. Leatherdale, and M. G. Bawendi, Science 287, 1011 (2000). 